\title{
Diagnostic evaluation of paediatric pulmonary hypertension in current clinical practice
}

\author{
Maurice Beghetti ${ }^{1}$, Rolf M.F. Berger², Ingram Schulze-Neick ${ }^{3}$, Ronald W. Day ${ }^{4}$, \\ Tomás Pulido ${ }^{5}$, Jeffrey Feinstein ${ }^{6}$, Robyn J. Barst ${ }^{7} \dagger$ and Tilman Humpl ${ }^{8}$ on behalf \\ of the TOPP registry investigators
}

\begin{abstract}
Affiliations: ${ }^{1}$ Paediatric Cardiology Unit, University Hospital, Geneva, Switzerland. ${ }^{2}$ Centre for Congenital Heart Diseases, Paediatric Cardiology, Beatrix Children's Hospital, University Medical Center Groningen, The Netherlands. ${ }^{3}$ Great Ormond Street Hospital for Children NHS Foundation Trust, London, UK. "University of Utah Health Care, Salt Lake City, UT, ${ }^{6}$ Stanford University Medical Centre, Palo Alto, CA, and ${ }^{7}$ Columbia University College of Physicians and Surgeons, New York, NY, USA. ${ }^{5}$ Cardiopulmonary Dept, National Heart Institute, Mexico City, Mexico. ${ }^{8}$ Critical Care Medicine and Cardiology, Hospital for Sick Children, University of Toronto, ON, Canada.
\end{abstract}

Correspondence: M. Beghetti, Paediatric Cardiology Unit, Children's Hospital, University Hospital of Geneva, 6 rue Willy Donzé 1211 Geneva 14, Switzerland. E-mail maurice.beghettiahcuge.ch

ABSTRACT Current paediatric pulmonary hypertension $(\mathrm{PH})$ diagnostic algorithms include some testing specifically for paediatrics, but it is unclear if this is used in clinical practice. We describe the current diagnostic workup of the TOPP (Tracking Outcomes and Practice in Paediatric Pulmonary hypertension) registry for suspected $\mathrm{PH}$.

We investigated 456 patients enrolled until February 2010. The majority had ECGs (94\%), echocardiograms (96\%) and/or chest radiographs (89\%) performed and these were the noninvasive tests most frequently used for evaluation of suspected PH. No patient had all three tests considered normal, suggesting the potential for the combined use to rule out $\mathrm{PH}$.

For evaluation of complications associated with heart catheterisation (HC) we analysed a total of 908 HCs reported until February 2012. Of these, 554 were at diagnosis and 354 in follow-up. Complications were reported in $5.9 \%$ with five deaths considered related to $\mathrm{HC}$, suggesting a higher rate of $\mathrm{HC}$ complications compared to adult studies. However, current recommendations support HC in paediatric $\mathrm{PH}$. A proper application of the risk/benefit ratio for $\mathrm{HC}$ requires further data. Most children did not undergo the diagnostic workup currently recommended for adults, which highlights either incomplete awareness of current guidelines and/or challenges their appropriateness for children.

@ERSpublications

Most PH children don't undergo full diagnostic evaluations; despite complications, catheterisation is used for diagnosis http://ow.ly/mx4GW

For editorial comments see page 580 .

Received: Sept 042013 | Accepted after revision: March 082013 | First published online: April 052013

Support statement: The TOPP registry is supported by an unrestricted research grant from Actelion Pharmaceuticals Ltd. Actelion does not participate in the management of the TOPP registry; neither does it have access to the database and the individual site and patient data. All decisions related to the Registry lie solely with the Executive Board Association for Paediatric Pulmonary Hypertension.

Conflict of interest: Disclosures can be found alongside the online version of this article at www.erj.ersjournals.com 


\section{Introduction}

Paediatric pulmonary hypertension $(\mathrm{PH})$ remains a serious medical condition with significant morbidity and mortality [1]. The challenges in paediatric $\mathrm{PH}$ are often more difficult than in adult $\mathrm{PH}$, due to lack of evidence-based guidelines for children and both greater procedure-related risk concerns in the small baby and drug-safety concerns in the growing child. Several single centre reports and multicentre paediatric registries have addressed treatments and outcomes [2-4]. Correct and timely diagnosis is important, as therapeutic decisions require the correct diagnosis and the early initiation of therapy may improve outcomes. The 2009 European guidelines for the diagnosis and treatment of adult $\mathrm{PH}$ have described in detail the diagnostic approach for $\mathrm{PH}$ in adults $[5,6]$. It also includes recommendations for paediatric patients: "the $\mathrm{PH}$ workup proposed for adults should also be considered in children" with a level of evidence $\mathrm{C}$ (consensus of opinion of experts and/or small studies, retrospective studies, registries) and class of recommendation IIA (weight of evidence/opinion is in favour of usefulness/efficacy).

Clinical presentation and aetiology in children is often different when compared to adults [7], despite a number of similarities in pathobiology and pathohistology. Pulmonary arterial hypertension (PAH) and Idiopathic/heritable PAH (IPAH/HPAH) associated with congenital heart disease account for at least $80 \%$ of the paediatric patients in large series $[7,2]$. In paediatric $\mathrm{PH}$ often more than one condition or comorbidity is present supporting the importance of a thorough diagnostic approach [8]. It is currently unclear if the workup in children with suspected $\mathrm{PH}$ should include all specific tests described in the adult workup to establish the correct diagnosis and detect all possible associated conditions and/or comorbidities. Prognostic parameters (e.g. including functional capacity, haemodynamic parameters), as well as specific paediatric risk and safety considerations, are crucial when developing an individualised treatment plan $[9,10]$. While current paediatric diagnostic algorithms consider some paediatric specificities, real-life practice remains unknown $[9,11,12]$.

Several questions are still unanswered in the paediatric population, e.g. in the current clinical practice: Which tests are used to screen for the detection of the disease? Which tests are used to identify aetiology for the disease? Which tests are used to evaluate the severity and the prognosis of the disease?

In this report we describe the diagnostic approach that was used in 456 children enrolled in TOPP (Tracking Outcomes and Practice in Paediatric Pulmonary hypertension) registry since January 2008, with newly (incident) and previously (prevalent) diagnosed PH patients included in the global TOPP registry. The data cut-off date was February 15, 2010 for the diagnosis workup and February 2012 for the heart catheterisation $(\mathrm{HC})$ related events.

\section{Methods}

Study design

The registry was designed as a multicentre, prospective, observational, cohort study and epidemiological data has been previously published [7]. Patients underwent clinical assessment and received treatment and follow-up according to the judgment of their local physician and were not on TOPP-related specific diagnostic and therapeutic protocols. The registry became operational in January 2008 and at the time the data was cut it included 31 centres from 19 countries.

To minimise the potential for selection bias, the protocol required all participating sites to screen consecutive patients who presented with either suspected or confirmed $\mathrm{PH}$ as an inpatient or outpatient at their centre. Patients who met the eligibility criteria were to be informed of the registry and invited to participate.

The study was designed and supervised by the Executive Advisory Board of the Association for Paediatric Pulmonary Hypertension. Data management and analyses were performed by a contract organisation working with the Executive Advisory Board. The protocol was approved by the relevant Institutional Review Boards and/or Ethics Committees.

Consecutive patients between the ages of 3 months and 18 years, at the time of diagnosis with $\mathrm{PH}$ confirmed by HC, were eligible for enrolment. All patients were diagnosed with PH on or after January 2001 in order to provide a study population representative of current clinical practice. This includes both newly diagnosed patients (incident, i.e. within 3 months of enrolment in the registry) and previously diagnosed patients (prevalent, i.e. $>3$ months prior to enrolment) were eligible.

Patients were eligible if they had PH belonging to Venice groups 1, 3, 4, or 5 (according to the Venice 2003 clinical classification [13], as this was the actual classification when the registry was designed) with the following specific groups: patients with congenital heart disease (CHD) with residual $\mathrm{PH}$ following surgery without residual left side obstruction (i.e. pulmonary capillary wedge pressure $\leqslant 12 \mathrm{mmHg}$ on $\mathrm{HC}>1$ year 
post repair) were eligible for enrolment and were included in the Venice PH group 1. The study did not include patients classified as Venice $\mathrm{PH}$ group 2.

The haemodynamic criteria for the diagnosis of $\mathrm{PH}$ were defined as a mean pulmonary arterial pressure $\geqslant 25 \mathrm{mmHg}$ at rest, pulmonary vascular resistance index (PVRI) to body surface area $>3$ Woods units $\cdot \mathrm{m}^{-2}$, and pulmonary capillary wedge pressure $\leqslant 12 \mathrm{mmHg}$. Consistent with current clinical practice, the rare patient without diagnostic HC could be enrolled in the TOPP registry if, under an independent review of the echocardiogram and/or histology report by the Executive Advisory Board, it was possible to validate the diagnosis and support the decision to abstain from the HC.

All diagnostic procedures were performed and judged "normal" or "abnormal" at the discretion of the local physician without any recommendations from the TOPP registry, to best reflect real-life clinical practice.

Due to the design of the TOPP registry, patients who died in relation to HC might not have been enrolled in the registry due to lack of haemodynamic data required for inclusion or due to the absence of informed consent. In order to minimise this bias and to increase robustness of HC-complication data, all HCs reported in the TOPP registry until February 2012 were collected and evaluated and all participating sites were additionally queried for procedure-related deaths or complications in this period. HC-related death was defined as death during induction of anaesthesia/sedation, during the procedure, within $24 \mathrm{~h}$ after the procedure, or related to any other HC-complications.

\section{Statistical methods and analysis}

The registry was designed to enrol a total of $\sim 450$ patients with at least a third as newly diagnosed patients and two-thirds as previously diagnosed patients. The protocol specified this a priori to ensure the appropriate number of incident patients would be enrolled, the ratio of prevalent to incident patients enrolled was monitored, and further enrolment of prevalent patients could be stopped once the target of two-thirds of the sample population or $\sim 300$ prevalent patients had been met.

A specific statistical analysis plan was developed to meet each of the registry objectives, and was finalised prior to conducting analyses. For the objectives of this report, the analyses are descriptive.

Continuous data were summarised using standard descriptive statistics, including mean, standard deviation, $95 \%$ confidence intervals, median, minimum, maximum and 25th and 75th percentiles, where appropriate. Descriptive summaries to compare the tests performed by year were produced to investigate possible changes in diagnostic approach over the period of diagnosis.

Categorical data were summarised using counts and percentages. Unless specified otherwise, the denominator for percentages was the total number of patients in each group. Missing data were not imputed. The 95\% confidence intervals were calculated using the normal approximation for the binomial distribution. Analyses were performed using SAS version 8.2 or higher (SAS Institute, Cary, NC, USA). Fisher's exact test (2-sided) was used to compare diagnostic test by prevalent/incident groups, age groups and aetiology groups. Fisher's exact test was also used to compare the number of patients with significant complications following $\mathrm{HC}$ in incident versus prevalent patients.

\section{Results}

The diagnostic procedures aimed at diagnosing/confirming $\mathrm{PH}$ are summarised in table 1 . The diagnostic tests aimed at excluding/diagnosing associated causes are shown in table 2. The assessment for severity of $\mathrm{PH}$ is displayed in table 3, with the sub-analyses of $\mathrm{PH}$ aetiology shown in table 4, and division, by age groups, shown in table 5 .

\section{Diagnostic tests aiming at diagnosis/confirmation of $\mathrm{PH}$}

The most frequently reported tests performed were ECG in 94\% (abnormal in 84\%), chest radiographs in $89 \%$ (abnormal in 71\%) and echocardiograms in $96 \%$ (abnormal in 96\%) of the patients. None of the enrolled patients had all three tests reported as "normal", $3 \%$ had two tests reported as "normal" and $18 \%$ had one test reported as "normal" (table 1).

$98 \%$ of patients had a diagnostic HC (by inclusion criteria) with the remaining $2 \%$ of the patients included in the registry after review by the Executive Advisory Board of their echocardiogram and/or lung histology.

Heart catheterisation

The haemodynamic data at diagnosis are shown in table 6.

Mean (95\% CI) cardiac index was $4.0 \mathrm{~L} \cdot \mathrm{min}^{-1} \cdot \mathrm{m}^{-2}\left(3.5-4.5 \mathrm{~L} \cdot \mathrm{min}^{-1} \cdot \mathrm{m}^{-2}\right)$ with normal limits $2.5-$ $4.0 \mathrm{~L} \cdot \mathrm{min}^{-1} \cdot \mathrm{m}^{-2}$ [14]. PVRI was significantly increased, with a mean (95\% CI) value of 15.8 Wood 
TABLE 1 Diagnostic tests aiming at diagnosing/confirming pulmonary hypertension

\begin{tabular}{|c|c|c|c|c|}
\hline & Incident patients & Prevalent patients & All patients & p-value ${ }^{\#}$ \\
\hline Patients N & 135 & 321 & 456 & \\
\hline ECG & 131 (97) & 299 (93) & 430 (94) & 0.11 \\
\hline Abnormal & 116 (89) & $269(90)$ & 385 (90) & \\
\hline Chest radiograph & 126 (93) & $280(87)$ & 406 (89) & 0.07 \\
\hline Abnormal & $103(82)$ & $222(80)$ & $325(80)$ & \\
\hline Echocardiogram & 132 (98) & 307 (96) & $439(96)$ & 0.45 \\
\hline Abnormal & $132(100)$ & 304 (99) & 436 (99) & \\
\hline All three tests normal" & 0 & 0 & 0 & \\
\hline Two tests normal & $6(4)$ & $9(3)$ & $15(3)$ & \\
\hline One test normal" & $23(17)$ & $58(18)$ & $81(18)$ & \\
\hline Heart catheterisation ${ }^{+}$ & 131 (97) & $314(98)$ & 445 (98) & 0.74 \\
\hline
\end{tabular}

Data are presented as $\mathrm{n}(\%)$, unless otherwise stated. $\mathrm{N}$ : total number of patients; $\mathrm{n}$ : all patients who were confirmed to have undergone the procedure. Denominator for percentages is N (test performed) and number of patients with test within test (abnormal). \#: Fisher's exact test to compare incident versus prevalent for diagnostic procedure being performed. ": ECG, echocardiogram and chest radiograph; ${ }^{+}$: it was intended that all patients would undergo heart catheterisation; however, 11 patients had indications on their case-report form that pulmonary hypertension was not confirmed by heart catheterisation, 10 patients provided no further heart catheterisation data.

units $\cdot \mathrm{m}^{-2}\left(7-16.8\right.$ Wood units $\left.\cdot \mathrm{m}^{-2}\right)$. The pulmonary to systemic vascular resistance ratio was $0.84(8-0.9)$. Of the 435 patients who provided $\mathrm{HC}$ data at diagnosis, $\mathrm{HC}$ was performed under general anaesthesia in 224 $(52 \%)$ and with conscious sedation in $205(48 \%)$ patients.

Reported complications associated with $\mathrm{HC}$ at diagnosis for the patients enrolled in the registry at the data cut-off point of February 2012 are shown in table 7.

During this period, data for 908 HCs were collected in the registry, 554 at diagnosis and 354 in follow-up. Complications were documented in 5.9\% for both diagnostic and follow-up HC. In total, there were five HC-related deaths, two at diagnosis and three at follow-up (0.6\%). At diagnosis, complications were reported in 37 patients $(6.7 \%$ ) (table 7 ). Of these 37 patients the most frequent events were systemic hypotension (17), requirement of inotropic support was seen (14), and pulmonary hypertensive crisis was observed in (10) patients, unexpected intensive care admission in (seven), cardiac arrest (five) and arrhythmia requiring intervention (four). No significant differences in complications at diagnosis were seen between prevalent and incident patients $(\mathrm{p}=0.86)$.

\section{Diagnostic tests aiming at excluding/diagnosing associated conditions}

Lung function tests

Pulmonary function tests were performed in 122 (27\%) of patients, lung perfusion scintigraphy in 104 $(23 \%)$, overnight oxygen saturation (in room air or oxygen) in $129(28 \%)$ and complete sleep recording in $68(15 \%)$

Chest computed tomography (CT) was performed in $41 \%$ and magnetic resonance imaging (MRI) in $9 \%$.

Lung biopsies were reported in 21 patients (5\%) with no deaths directly related to the procedure, but with complications in seven patients (33\%). For three out of these patients, no information on complications could be obtained because the biopsy was performed in another centre that was not centre in which the patient was enrolled. For the remaining four patients the complications consisted of bleeding (three), prolonged intubation (two), $\mathrm{PH}$ crisis (one) shock (one) and sepsis (one) ( $>1$ event per patient). For five of the 21 patients with a lung biopsy, no information on whether or not the patient had complications could be obtained.

Significant differences existed between the PH Venice classification groups and between age groups, with respect of the different tests. The number of patients with overnight oxygen saturation performed was significantly different between group 3 (lung disease) and group 1 (PAH) CHD patients and group 1 IAPH $(\mathrm{p}<0.001)$, and occurred more frequently in patients diagnosed as group 3 (table 4$)$. In contrast, pulmonary function tests were performed more often in patients with IAPH or associated forms of PAH compared to those diagnosed as group 3, but the age of the patient plays a role as pulmonary function tests cannot be performed in younger patients.

Lung perfusion scintigraphy was performed in $23 \%$ of the patients, more in IAPH and non-CHD associated $\mathrm{PAH}(<0.001)$. Lung perfusion scintigraphy, chest CT and MRI were performed predominantly in patients 
TABLE 2 Diagnostic tests aimed at excluding/diagnosing associate conditions

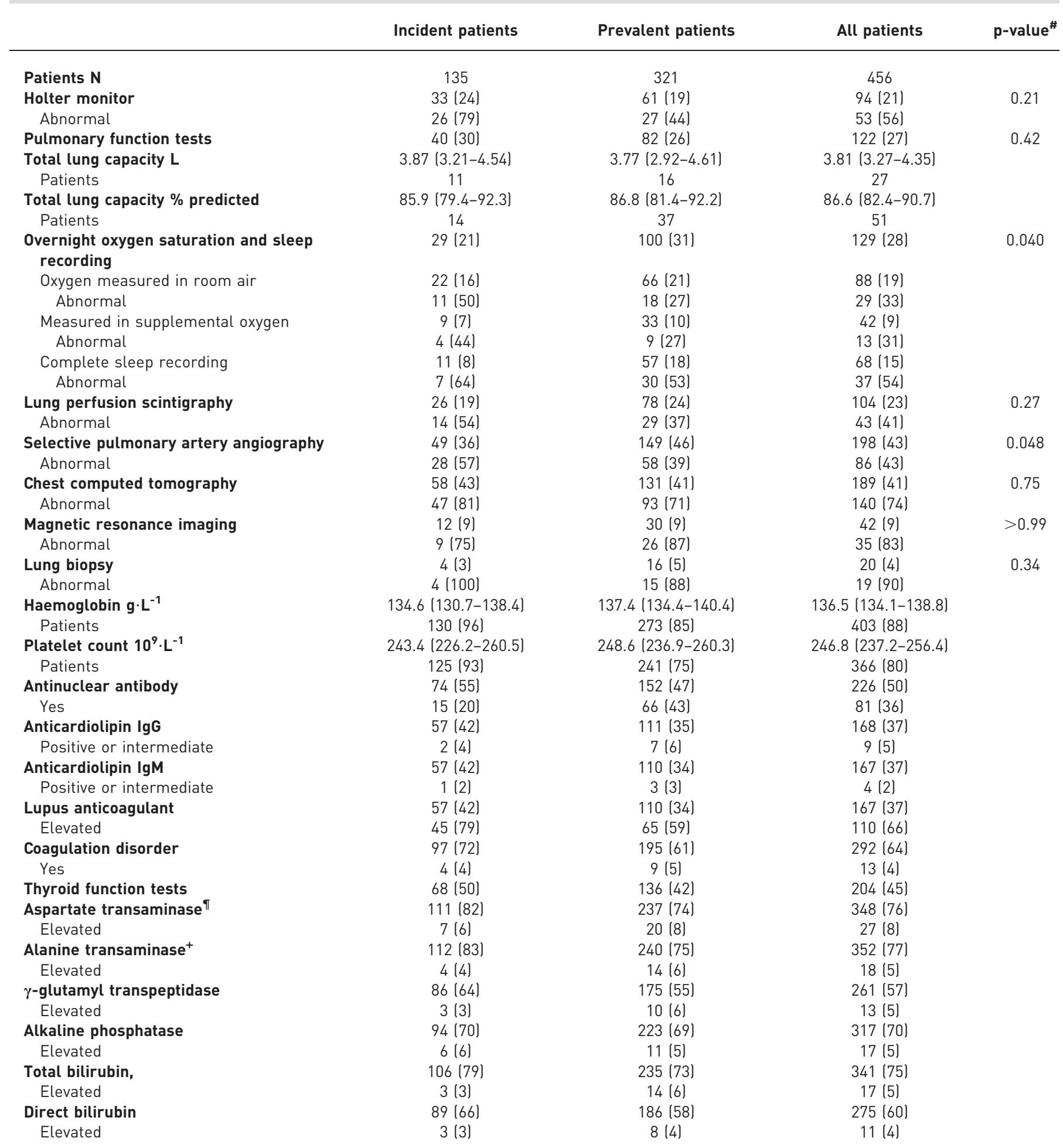

Data are presented as $\mathrm{n}(\%)$, mean $(95 \% \mathrm{Cl})$ or $\mathrm{n}$, unless otherwise stated. $\mathrm{N}$ : total number of patients; $\mathrm{n}$ : all patients who were confirmed as having undergone the procedure; Ig: immunoglobulin. Denominator for percentages is $\mathrm{N}$ for the test performed rows and the number of subjects with the test performed for the results rows. " : Fisher's exact test to compare incident versus prevalent for diagnostic procedure being performed; " ${ }^{\text {": also }}$ known as serum glutamic-oxaloacetic transaminase; ${ }^{+}$: also known as serum glutamic-pyruvic transaminase. 
TABLE 3 Assessment of severity

Incident patients

Prevalent patients

All patients

p-value ${ }^{\#}$

Patients $\mathrm{N}$

6-min walk test

6-min walking distance $\mathrm{m}$

Cardiopulmonary exercise testing

Patients

Peak $V^{\prime} \mathrm{O}_{2}, \mathrm{n}$

$\%$ predicted

B-type natriuretic peptide $\mathrm{ng} \cdot \mathrm{L}^{-1}$

Patients

$\mathrm{N}$-terminal b-type natriuretic peptide $\mathrm{ng} \cdot \mathrm{L}^{-1}$

Patients

Uric acid $\mu \mathrm{mol} \cdot \mathrm{L}^{-1}$

Patients

Troponin

Elevated

C-reactive protein $\mathrm{mg} \cdot \mathrm{L}^{-1}$

Patients
135

$51(38)$

$438.9(408.8-469.0)$

$5(4)$

$58.0(28.1-87.9)$

$298.7(115.7-481.7)$

31 (23)

4205 (953-7457)

33 (24)

317.1 (288.3-345.9)

61 (45)

26 (19)

3 (12)

$7.8(1.6-14.1)$

$68(50)$
321

124 (39)

$394.2(371.3-417.0)$

29 (9)

28

$50.6(42.6-58.7)$

$337.3(177.1-497.6)$

$66(21)$

4417 (2702-6131)

62 (19)

317.6 (297.0-338.3)

108 (34)

38 (12)

$3(8)$

$5.2(3.9-6.5)$

$137(43)$
456

175 (38)

$407.2(388.7-425.7)$

$34(7)$

33

$51.8(44.3-59.2)$

325.0 (203.1-446.9)

97 (21)

4343 (2788-5898)

95 (21)

$317.4(300.8-334.0)$

169 (37)

$64(14)$

6 (9)

$6.1(3.8-8.3)$

$205(45)$

Data are presented as $\mathrm{n}(\%)$ or mean $(95 \% \mathrm{Cl})$, unless otherwise state. $\mathrm{N}$ : total number of patients; $\mathrm{n}$ : all patients who were confirmed as having undergone the procedure. Denominator for percentages was $\mathrm{N}$ for the test-performed rows and the number of subjects with the test performed for the results rows.

diagnosed as IPAH. There is a nonsignificant increase that these tests were performed more frequently with increasing age.

Laboratory tests

Data on a number of laboratory measurements are shown in table 2. Reporting of laboratory tests appears

to be incomplete.

TABLE 4 Test performed at diagnosis by diagnostic groups

All IPAH/FPAH

Group 1 PAH

Group 3 PH p-value

patients

APAH non-CHD

\begin{tabular}{llll}
\multicolumn{3}{c}{ APAH-CHD } \\
\hline \multirow{2}{*}{ All CHD } & \multicolumn{3}{c}{ Shunt } \\
\cline { 2 - 4 } & $\begin{array}{c}\text { Unrepaired/ } \\
\text { partial repair }\end{array}$ & Repaired & Never \\
\end{tabular}

\begin{tabular}{|c|c|c|c|c|c|c|c|c|c|}
\hline Patients N & 456 & 212 & 26 & 160 & 91 & 57 & 12 & 52 & \\
\hline ECG & 430 (94) & & & 148 (93) & $86(95)$ & $50(88)$ & $12(100)$ & 49 (94) & 0.06 \\
\hline Chest radiograph & 406 (89) & 195 (92) & 24 (92) & $138(86)$ & $77(85)$ & $49(86)$ & 12 (100) & $46(88)$ & 0.05 \\
\hline Echocardiogram & $439(96)$ & 205 (97) & $26(100)$ & $154(96)$ & 90 (99) & $52(91)$ & $12(100)$ & $50(96)$ & 0.21 \\
\hline $\mathrm{HC}^{\circ}$ & 445 (98) & 208 (98) & $24(92)$ & 158 (99) & $91(100)$ & $55(96)$ & $12(100)$ & $50(96)$ & 0.40 \\
\hline Holter monitor & $94(21)$ & 49 (23) & $5(19)$ & $38(24)$ & $18(20)$ & 19 (33) & 1 (8) & $2(4)$ & 0.001 \\
\hline Laboratory function tests & 411 (90) & 191 (90) & $23(88)$ & 148 (93) & 85 (93) & $51(89)$ & 12 (100) & $46(88)$ & 0.42 \\
\hline 6-min walk test & 175 (38) & 93 (44) & $11(42)$ & 60 (38) & $38(42)$ & $21(37)$ & $1(8)$ & 10 (19) & 0.003 \\
\hline Cardiopulmonary exercise test & 34 (7) & $16(8)$ & $4(15)$ & $7(4)$ & $3(3)$ & $4(7)$ & 0 & $6(12)$ & 0.17 \\
\hline Pulmonary function tests & 122 (27) & $67(32)$ & $13(50)$ & 31 (19) & $16(18)$ & $13(23)$ & $2(17)$ & $9(17)$ & 0.008 \\
\hline $\begin{array}{l}\text { Overnight oxygen saturation and } \\
\text { sleep recording }\end{array}$ & $129(28)$ & $62(29)$ & 9 (35) & 31 (19) & $13(14)$ & $17(30)$ & $1(8)$ & $25(48)$ & $<0.001$ \\
\hline Lung perfusion scintigraphy & 104 (23) & 69 (33) & $8(31)$ & $16(10)$ & $7(8)$ & $9(16)$ & 0 & 10 (19) & $<0.001$ \\
\hline Pulmonary angiography & $198(43)$ & $102(48)$ & $11(42)$ & 49 (31) & $24(26)$ & 22 (39) & $3(25)$ & $33(63)$ & $<0.001$ \\
\hline Chest computed tomography & $189(41)$ & $102(48)$ & $20(77)$ & 36 (23) & $15(16)$ & $17(30)$ & $4(33)$ & $27(52)$ & $<0.001$ \\
\hline Magnetic resonance imaging & 42 (9) & $29(14)$ & $4(15)$ & $7(4)$ & $4(4)$ & $2(4)$ & $1(8)$ & $1(2)$ & 0.001 \\
\hline Lung biopsy & $21(5)$ & $8(4)$ & $1(4)$ & $9(6)$ & $4(4)$ & 5 (9) & 0 & $3(6)$ & 0.62 \\
\hline
\end{tabular}

Data are presented as $\mathrm{n}(\%)$, unless otherwise stated. N: total number of patients; $\mathrm{n}$ : all patients who were confirmed as having undergone the procedure; PAH: pulmonary arterial hypertension; IPAH: idiopathic PAH; FPAH: familial PAH; APAH: associated PAH; CHD: congenital heart disease; PH: pulmonary hypertension; HC: heart catheterisation. For missing data, it was assumed that the procedure was not performed. \#: Fisher's exact test to compare group 3 PH versus all-CHD versus IPAH/FPAH for diagnostic procedure being performed; ${ }^{\circ}$ : it was intended that all patients would undergo HC; however, 11 patients had indications on their case-report form that PH was not confirmed by $\mathrm{HC}$, 10 patients provided no further HC data. Denominator for percentages is the number of subjects in the population in each subgroup (n). 
TABLE 5 Test performed at diagnosis by age groups

Patient age

\begin{tabular}{|c|c|c|c|c|c|c|}
\hline & & & \\
\hline & $3 \leqslant 24$ months & $2-6$ years & $7-11$ years & $12-18$ years & & \\
\hline Patients ${ }^{\top} \mathrm{N}$ & 94 & 134 & 108 & 118 & 456 & \\
\hline ECG & $90(96)$ & $127(95)$ & $102(94)$ & $111(94)$ & $430(94)$ & 0.42 \\
\hline Echocardiogram & $88(94)$ & $129(96)$ & 106 (98) & 116 (98) & $439(96)$ & 0.58 \\
\hline $\mathrm{HC}^{+}$ & $91(97)$ & 132 (99) & 103 (95) & 117 (99) & 445 (98) & 0.25 \\
\hline Holter monitor & $9(10)$ & $26(19)$ & $21(19)$ & $38(32)$ & $94(21)$ & 0.001 \\
\hline Cardiopulmonary exercise test & 0 & $3(2)$ & $12(11)$ & $19(16)$ & 34 (7) & $<0.001$ \\
\hline Pulmonary function tests & $2(2)$ & $16(12)$ & 40 (37) & $64(54)$ & $122(27)$ & $<0.001$ \\
\hline $\begin{array}{l}\text { Overnight oxygen saturation } \\
\text { and sleep recording }\end{array}$ & 31 (33) & $45(34)$ & $23(21)$ & $30(25)$ & $129(28)$ & 0.10 \\
\hline Lung perfusion scintigraphy & $11(12)$ & $27(20)$ & $28(26)$ & $38(32)$ & 104 (23) & 0.004 \\
\hline Pulmonary angiography & $42(45)$ & $61(46)$ & $45(42)$ & $50(42)$ & $198(43)$ & 0.89 \\
\hline
\end{tabular}

Data are presented as $\mathrm{n}(\%)$ unless otherwise stated. $\mathrm{N}$ : total number of patients; $\mathrm{n}$ : all patients who were confirmed as having undergone the procedure. HC: heart catheterisation. \#: Fisher's exact test to compare across the age groups for diagnostic procedure being performed; ": the exact date of birth could not be obtained for two patients; however, both were in the paediatric age group and included in the general analysis and do not appear in this table; ${ }^{+}$it was intended that all patients would undergo HC; however, 11 patients had indications on their case-report form that pulmonary hypertension was not confirmed by HC, 10 patients provided no further HC data. ${ }^{\S}$ : nine of these patients $(2-6$ years $n=2 ; 7-11$ years $\mathrm{n}=1 ; 12-8$ years $\mathrm{n}=6$ ) have Down syndrome or other chromosomal abnormalities. The denominator for percentages was $\mathrm{N}$.

Screening for coagulation disorders was performed in $64 \%$ of the patients, screening for connective tissues disease was performed in 50\%, thyroid function tests in $45 \%$ and liver function tests in $79 \%$.

\section{Assessment of severity}

Haemodynamic data have been presented in the HC section.

Tests to assess disease severity or prognostic parameters, such as b-type natriuretic peptide (BNP), N-terminal pro $\mathrm{BNP}$, troponin and uric acid were performed in $21 \%, 21 \%, 14 \%$ and $37 \%$ of patients, respectively.

\section{TABLE 6 Haemodynamic parameters}

\begin{tabular}{|c|c|c|c|}
\hline & Incident patients & Prevalent patients & All patients \\
\hline Patients N & 135 & 321 & 456 \\
\hline Mean pulmonary arterial pressure $\mathrm{mmHg}$ & $57.7(54.0-61.4)$ & $57.2(55.2-59.2)$ & $57.4(55.6-59.1)$ \\
\hline Pulmonary vascular resistance index Wood units $\cdot \mathrm{m}^{-2}$ & $15.9(13.7-18.1)$ & $15.7(14.5-16.9)$ & $15.8(14.7-16.8)$ \\
\hline Patients & 99 & 270 & 369 \\
\hline Cardiac index $\mathrm{L} \cdot \mathrm{min}^{-1} \cdot \mathrm{m}^{-2}$ & $3.6(3.3-4.0)$ & $4.1(3.5-4.7)$ & $4.0(3.5-4.5)$ \\
\hline Patients & 92 & 260 & 352 \\
\hline $\mathrm{SvO}_{2} \%$ & $64.0(61.2-66.7)$ & $66.5(65.0-67.9)$ & $65.7(64.4-67.0)$ \\
\hline Patients & 79 & 188 & 267 \\
\hline Pulmonary capillary wedge pressure $\mathrm{mmHg}$ & $8.8(8.3-9.2)$ & $8.4(8.1-8.7)$ & $8.5(8.3-8.8)$ \\
\hline Patients & 127 & 300 & 427 \\
\hline
\end{tabular}

Data are shown as mean $(95 \% \mathrm{Cl})$ or $\mathrm{n}(\%)$, unless otherwise stated. $\mathrm{SvO}_{2}$ : mixed venous oxygen saturation. 
TABLE 7 Heart catheterisation (HC) complications at diagnosis

\begin{tabular}{|c|c|c|c|c|}
\hline & Incident patients & Prevalent patients & All patients & p-value ${ }^{\#}$ \\
\hline Patients N & 244 & 324 & 568 & \\
\hline $\mathrm{HC}^{\circ}$ & $238(98)$ & $316(98)$ & 554 (98) & \\
\hline Patients with significant complications during/after HC & $15(6)$ & $22(7)$ & $37(7)$ & 0.86 \\
\hline Hypotension requiring intervention & $8(3)$ & $9(3)$ & $17(3)$ & \\
\hline Inotropic support required & 7 (3) & $7(2)$ & $14(3)$ & \\
\hline PH crisis & 7 (3) & $3(<1)$ & $10(2)$ & \\
\hline Arrhythmia requiring intervention & $1(<1)$ & $3(<1)$ & $4(<1)$ & \\
\hline Unexpected ICU admission after $\mathrm{HC}$ & $4(2)$ & $3(<1)$ & $7(1)$ & \\
\hline Cardiac arrest & $4(2)$ & $1(<1)$ & $5(<1)$ & \\
\hline Other & 0 & $5(2)$ & $5(<1)$ & \\
\hline Pulmonary haemorrhage & 0 & $1(<1)$ & $1(<1)$ & \\
\hline $\begin{array}{l}\text { Death }{ }^{+} / \text {stroke/haemothorax/pneumothorax/pericardial } \\
\text { effusion/cardiac perforation/bleeding requiring transfusion }\end{array}$ & 0 & 0 & 0 & \\
\hline Peri-HC complications recorded as reason for death ${ }^{\S}$ & $2(<1)$ & 0 & $2(<1)$ & \\
\hline
\end{tabular}

Data are presented as $\mathrm{n}(\%)$ unless otherwise stated. ICU: intensive care unit. " ${ }^{\text {: }}$ Fisher's exact test to compare incident versus prevalent. " : it was intended that all patients would undergo HC; however, 11 patients had indications on their case-report form that pulmonary hypertension (PH) was not confirmed by HC, 10 patients provided no further $\mathrm{HC}$ data; ${ }^{+}$: death during procedure or within $24 \mathrm{~h}$ (recorded as a complication in the case report form; ${ }^{\S}$ : deaths relating to $\mathrm{HC}$ at diagnosis are presented in the table, of note, one of these patients did not undergo $\mathrm{HC}$ and died whilst being anesthetised in preparation for the HC. Three further patients (all prevalent) had "peri-HC complications" recorded as the reason for death following follow-up HCs. For number of patients with $\mathrm{HC}$ and number of patients with peri-HC complications recorded as reason for death the denominator for percentages is $\mathrm{N}$. All other percentages were based on the number of patients undergoing HC as the denominator ( $\mathrm{n}$ ).

\section{Exercise capacity}

Assessment of exercise capacity using 6-min walk test (6MWT) and cardiopulmonary exercise testing (CPET) was reported in $38 \%$ and $7 \%$ of the patients, respectively. The $6 \mathrm{MWT}$ was performed less in patients with Venice group 3 compared to other groups, but these patients were also younger. In patients aged $>12$ years, $71 \%$ had 6 MWT data reported. CPET was performed significantly less overall and performed in only three patients aged $<7$ years.

\section{IPAH}

Although IPAH and PH group 3 patients had the most complete workup compared to the other forms, the majority did not have what is suggested in adult guidelines or paediatric recommendations. Of the 212 IPAH patients, 208 had a chest radiograph, ECG or echocardiography performed and 191 had all three. In these 208 children, none had all three tests reported as "normal" but 139 had all three tests reported as "abnormal". Among the 212, 67 had lung function tests performed that were reported as "normal", 178 had liver function tests to evaluate hepatic function, 191 had laboratory tests, 15 had laboratory tests looking for a connective tissue disease, and 157 had a coagulation workup to assess for coagulation disorders.

Of the 145 IAPH patients, who did not have pulmonary function tests, 65 had a chest CT and 30 had oxygen saturation recordings to evaluate for lung disease. 115 had liver workup, 33 had laboratory tests for connective tissue disease and 98 for coagulation disorders.

Of the total IPAH cohort $(n=212)$, in 69 patients $(33 \%)$ a lung perfusion scintigraphy had been performed.

As patients were diagnosed over a 9-year period, the percentage of patients that had each of the tests by year of diagnosis was calculated to see if there was a change in diagnostic approach over time (data not shown). There were no differences observed in any of the tests comparing prevalent and incident patients over the last 10 years, suggesting there were no changes in the diagnostic approach over the period of diagnosis. No major differences were seen except for a decrease in the number of lung biopsies (overall only 5\%).

\section{Discussion}

The analysis of diagnostic evaluation performed in patients included in the TOPP registry shows first that ECGs, chest radiographs and echocardiograms are collectively good screening tests for the detection of $\mathrm{PH}$ in children. Secondly, that incomplete testing is performed for the identification of causes of PH. Thirdly, incomplete testing of functional capacity is performed for evaluation of severity of the disease. Finally, the rate of $\mathrm{HC}$ complications is higher than in adults. 
One of the most significant findings of this study is that in real-life practice the majority of the paediatric patients considered to have $\mathrm{PH}$ by their physician (at a paediatric $\mathrm{PH}$ centre) did not undergo a detailed and complete diagnostic workup as recommended in the adult guidelines [5,6]. This illustrates the complexity applying guidelines in clinical practice and also that extrapolation from adults is not straightforward. Paediatric $\mathrm{PH}$ shows some differences compared to adult $\mathrm{PH}$, which may be the underlying reason for the diagnostic approach used by experienced physicians. Certainly, this may be due to the difficulty of performing some tests in children requiring either the cooperation of the young patient or performing a procedure considered too high risk in relation to the value of obtained data and clinical status (i.e. HC, MRI or lung biopsy). Paediatricians routinely discuss the risk benefit of procedures that may have influenced the results. Another potential factor may be the adaptation of the diagnostic approach to the "suspected" underlying disease, i.e. the avoidance of clearly non-contributory tests in view of an obvious diagnosis. This seems to be true for patients with "definite" lung disease or CHD where all tests allowing exclusion of other forms of $\mathrm{PH}$ were not performed. Registry data cannot prove the under-diagnosis of some aetiologies or over-diagnosis of IPAH; a prospective study would be more useful to assess whether this is true. However, this has been alluded to in single-centre reports where multiple causes or aetiologies are often diagnosed in paediatric patients when the complete workup is performed [8].

Specifically, the present study demonstrates that most of the patients enrolled with the diagnosis of IPAH do not have the complete workup recommended in the European adult guidelines or in the current paediatric algorithms to rule out other causes of $\mathrm{PH}[9,11]$. This is particularly true for omitting lung function tests, workup for connective tissue disease and coagulation disorders. Lung scintigraphy was performed in a very small number of patients raising the question of under-diagnosis of Venice group 4 patients with chronic thromboembolic pulmonary hypertension $(\mathrm{CTEPH})$ Although CTEPH is rare in children, it is a potential cause of paediatric $\mathrm{PH}$ and necessitates appropriate evaluation. MADANI et al [15] reported 17 paediatric CTEPH cases over an 11-year period requiring thromboendarterectomy surgery.

Lung biopsy was only performed in $5 \%$ of the patients with no deaths and a $33 \%$ rate of complications, which compares to previous reports. In a large series of 64 biopsies performed in 58 patients (18 patients with PH) from 1976 to 1996 [16], only one out of 19 deaths was related to the lung biopsy itself (patient with severe $\mathrm{PH}$ ). However, the procedure was associated with a $30 \%$ hospital mortality and $11 \%$ morbidity rate. 13 patients died from their disease and five had support withdrawn. It is essential to outweigh the risk of lung biopsy in this population and carefully address the potential benefit of this procedure, in particular with regards to the consequences for the treatment strategy.

The findings of this registry trigger the discussion whether or not to perform a complete workup in paediatric patients. As recent studies have shown that paediatric patients may present with different associated conditions causing $\mathrm{PH}$ [4], it is strongly recommended to perform a complete workup. However, the specific risk and safety issues in paediatric practice, as well as the significance and relevance of the test performed in experienced centres, needs to be recognised. This may necessitate an adapted approach in some patients and needs to be carefully tailored so that precise aetiology, necessary to establish the specific paediatric therapeutic approaches, will not be missed.

For comparison, a look to the application of the diagnostic algorithm in the real-life adult practice may be useful. The European adult guidelines suggest following a diagnostic algorithm but from current published adult registry data accounting for the real clinical practice [17-24], it is difficult to find data on the diagnostic procedures performed, as most of the discussions are focusing on hemodynamic results. THENAPPAN et al. [24] reported for a US registry that only $67 \%$ of patients had antinuclear antibody testing, $50 \%$ had a CT scan and $72 \%$ lung scintigraphy. Exercise capacity tolerance was only reported in $57 \%$ and HC in $90 \%$. Similarly, in the French registry only $82 \%$ of the patients had $6 \mathrm{MWT}$ and $96 \%$ HC [22]. This also indicates that in adult practice a complete workup may not always be performed.

\section{Diagnostic tests aiming at diagnosis/confirmation of $\mathrm{PH}$}

There was a $100 \%$ likelihood of having at least one of the screening tests (ECG, echocardiogram or chest radiograph) reported as "abnormal" by the local physician leading to HC and confirmation of PH. However, registry data are not suited to confirm that the combination of a normal ECG, echocardiogram and chest radiograph definitely rule out $\mathrm{PH}$. It was not assessed why a test was considered as abnormal. This is a limitation as abnormal may not be considered always as correlated with PH. Based on these data we would recommend to use these three noninvasive tests in combination as screening for suspected $\mathrm{PH}$ in children.

Heart catheterisation

Reported HC complications (diagnostic and follow-up HCs) showed a $0.6 \%$ rate of procedure-related death and $5.9 \%$ rate of complications. The rate of complications in this study may be underestimated, due to the 
design of the TOPP registry requiring full, invasive, haemodynamic evaluation and informed consent. Thus, our data illustrate that invasive haemodynamic testing is not without the potential for serious complications in paediatrics.

TAYLOR et al [25] reported in a series of 75 consecutive children with confirmed PH (HC performed between 1999 and 2004) four cardiac arrests requiring cardiopulmonary resuscitation and one death, accounting for a $6 \%$ rate of cardiac arrest and/or death. The same centre taking part in this TOPP registry confirmed that no deaths or major complications were reported from 2008 to 2011, probably as a result of detailed procedure changes (unpublished data). However, in the overall TOPP cohort, no significant differences were seen in prevalent and incident patients suggestive of no change in the procedural risk with newly diagnosed patients. CARMOSINO et al. [26] reviewed peri-procedural complications in paediatric $\mathrm{PH}$ patients with a reported 5\% rate of major HC complications and a 1.4\% mortality rate. A recent USA survey in children undergoing HC reported few adverse events (3.9\%) with no procedural deaths in 217 patients [27]. The risk of adverse events was higher for patients with supra-systemic baseline pulmonary arterial pressures. No significant differences were seen in prevalent and incident patients suggestive of no higher procedural risk in newly diagnosed patients. Centres participating in the TOPP registry are considered to be recognised $\mathrm{PH}$ centres and, as such, may be considered as experienced centres to perform HC procedures in these patients. Nevertheless, the HC-complication rate in paediatric $\mathrm{PH}$ patients seems to be higher than reported in adults with $\mathrm{PH}$, although differences in definitions of complications and in inclusion criteria requires restraints in direct comparisons [28].

However, the recent scientific statement of the American Heart Association, on the indications for HC in paediatric cardiac disease, still consider that $\mathrm{HC}$ is recommended to assess pulmonary vascular resistance and reversibility of $\mathrm{PH}$ in patients with $\mathrm{CHD}$ or IPAH when accurate assessment of pulmonary vascular resistance is needed to make a surgical or medical decision with a level or evidence B (Data derived from a single, randomised trial or nonrandomised studies) [29]. This is also supported by MulLEN et al. [11], who recommend $\mathrm{HC}$ in the acute assessment of paediatric $\mathrm{PH}$ to confirm the diagnosis and establish severity with a class 1 (condition for which there is evidence and/or agreement that a given procedure is beneficial, useful and effective) and a level of evidence $\mathrm{C}$ (only consensus opinion of experts case studies or standard of care). It was also noted that $\mathrm{HC}$ with vasodilator testing is recommended to guide initial therapy (class 1 level of evidence B).

Exercise capacity assessments

The use of the other tests seems to be driven more by age of patients, as particular tests requiring cooperation or sedation. This includes exercise testing with almost no patients aged $<7$ years having exercise tests. In older patients exercise capacity assessments were not performed in all patients, which may be related to a lack of developmental ability (such as in children with trisomy 21) or other reasons not reported.

This raises the question of the utility of these tests in children and their use as an endpoint in clinical trials. LAMMERS et al. [30] reported utility of 6MWT in children with significant limitations in exercise endurance. In the largest paediatric $\mathrm{PH}$ trial, CPET was used as a primary endpoint, but could only be performed $<50 \%$ of the recruited patients because of young age or lack of developmental ability to exercise reliably [31]. Taking this into account, it is important to note that both tests require specific expertise in the paediatric population, both for performing the tests and in analysing the data and can be very helpful in assessing prognosis and evaluating response to therapy [32, 33].

There were no differences observed in any of the tests comparing prevalent and incident patients over the last 10 years. Descriptive summaries of the number of patients undergoing each test serially, year per year, did not reveal that any major changes occurred in the diagnostic approach in patients diagnosed before 2008 and those diagnosed between 2008 and 2010. This finding is both interesting and concerning, as new guidelines with more detailed diagnostic algorithm have been implemented to increase education and awareness of $\mathrm{PH}$ in children. However, in contrast, a change in clinical practice from a diagnostic evaluation standpoint has not been noticeable in children with suspected of PH when comparing long-standing prevalent patients with recently diagnosed incident ones.

\section{Limitations}

Selection of centres participating in the TOPP registry was based on the known experience of these centres in diagnosing and treating paediatric $\mathrm{PH}$. This may have created a selection bias, as not being representative of all centres taking care of paediatric $\mathrm{PH}$ patients. The diagnostic approach was at the decision of the local physician and the validity of any diagnostic algorithm used by the centres cannot be evaluated. As such sensitivity, specificity, positive or negative-predictive value of the tests cannot be assessed, because only 
patients who had the confirmation of the $\mathrm{PH}$ by $\mathrm{HC}$ were included. And lastly, HC complications may have been underreported as patients with serious HC complications may have not been enrolled due to the design of the registry.

\section{Conclusions}

There is currently no consensus on a diagnostic approach of children with suspected $\mathrm{PH}$. However, the results from this large paediatric PH registry, suggest that chest radiographs, ECGs and echocardiogram are good screening tools in the paediatric population with the advantages of being relatively cost-effective, noninvasive and accessible. The HC related complication rate is higher than in adults and the risks of this procedure has to be weighed against the value of invasive hemodynamic data in tailoring individualised treatment strategies in paediatric PH. The TOPP-registry data are unique in providing a real-world experience including centres from developing and developed countries. Based on these data, the real-world practice does not appear to be following the current recommendations of complete workup of paediatric patients suspected with $\mathrm{PH}$, but this may also be true for adults.

Unfortunately, exercise capacity, the most frequently used test addressing the patient's functional status and response to treatment for the adult patient, cannot be performed in many paediatric patients. Even if they are aged $>6$ years, many cannot exercise reliably due to comorbid conditions such as Trisomy 21 .

We recommend adapting the guidelines to the paediatric population, despite the difficulties to perform some tests in this population. Only by doing so, we will be able to have appropriate data on how to best assess children with $\mathrm{PH}$.

The development of an adaptive design for a specific diagnostic algorithm for paediatric $\mathrm{PH}$ patients is required taking into account the risks and benefits of the different procedures, and in particular HC. The TOPP-registry data should help in the development of such an algorithm that could then be assessed prospectively.

\section{Acknowledgements}

We thank Katherine Hutchinson and Emily Wood of Quanticate, Hitchin, UK for statistical analysis and Rita Locher (Project Manager; IBR ag, Reinach, Switzerland) for continuous organisational support.

The TOPP registry investigators, listed in country, alphabetical, order (excluding current authors), are: R. Weintraub and I. King, Royal Children's Hospital, Dept of Cardiology, Melbourne, Australia; R. Geiger, Innsbruck Medical University, Paediatric Cardiology, Innsbruck, Austria; S. Mattos, Royal Portuguese Hospital, Maternal-Fetal Cardiac Unit, Recife, Brazil; Z.C. Jing and Xin Jiang, Dept of Cardio-Pulmonary Circulation, Shanghai Pulmonary Hospital, Tongji University School of Medicine, Shanghai, China; L.Sondergaard, Rigshospitalet, University of Copenhagen, Heart Center, Copenhagen; Denmark; D. Bonnet and M. Levy, Paediatric Cardiology, Université Paris Descartes, Sorbonne Paris Cité, AP-HP, Necker Enfants Malades, Paris, France; S. Mebus, Deutsches Herzzentrum München, Technische Universität München, Germany; Ch. Apitz, Kinderherzzentrum, Giessen, Germany; A. Szatmari, Hungarian Institute of Cardiology, Paediatric Cardiac Center, Budapest, Hungary; O. Milanesi, Universita di Padova, Dipartimento di Pediatria, Padova, Italy; T. Saji, Toho University Medical Center Omori Hospital, Division of Paediatric Cardiology, Tokyo, Japan; M.T.R. Roofthooft, Centre for Congenital Heart Diseases, Paediatric Cardiology, Beatrix Children's Hospital, University Medical Center Groningen, The Netherlands; P. de la Garza, National Heart Institute, Mexico City, Mexico; H. Brun, Rikshospitalet, Section for Paediatric Cardiology, Oslo, Norway; L. Moll, Polish Mothers Hospital Research Institute, Lodz, and M. Zuk, Children's Memorial Health Institute, Dept of Cardiology, Warsaw, Poland; M. Fasnacht Boillat, Universitäts Kinderklinik, Zurich, Switzerland; R. Olgunturk and S. Kula, Gazi Universitesi Tip Fakultesi, Pediyatrik Kardiyoloji ABD, Ankara, and D. Alehan, Hacettepe University Ihsan Dogramaci, Tip Fak. Pediyatrik Kardiyoloji, Ankara, Turkey; J.T. Berger, Children's National Medical Center, Critical Care, Washington, WA, D. Dunbar Ivy and K. Miller Reed, Children's Hospital Colorado, Aurora, CA, A. Atz Charleston, SC, G.B. Mallory, Texas Children's Hospital, Baylor College of Medicine, Houston, TX, J. D. Moore and E.D. Austin, Vanderbilt Children's Hospital, Divisions of Cardiology and Pulmonary Medicine, Nashville, TN, M. Ogawa, Stanford University Medical Center, Palo Alto, CA, and D. Yung, Seattle Children's Hospital, Seattle, WA, USA.

\section{References}

1 Barst RJ, Ertel SI, Beghetti M, et al. Pulmonary arterial hypertension: a comparison between children and adults. Eur Respir J 2011; 37: 665-677.

2 Barst RJ, McGoon MD, Elliott CG, et al. Survival in childhood pulmonary arterial hypertension: insights from the registry to evaluate early and long-term pulmonary arterial hypertension disease management. Circulation 2012; 125: 113-122.

3 Moledina S, Hislop AA, Foster H, et al. Childhood idiopathic pulmonary arterial hypertension: a national cohort study. Heart 2010; 96: 1401-1406.

4 van Loon RL, Roofthooft MT, Hillege HL, et al. Pediatric pulmonary hypertension in the Netherlands: epidemiology and characterization during the period 1991 to 2005. Circulation 2011; 124: 1755-1764.

5 Galiè N, Hoeper MM, Humbert M, et al. Guidelines for the diagnosis and treatment of pulmonary hypertension: the Task Force for the Diagnosis and Treatment of Pulmonary Hypertension of the European Society of Cardiology (ESC) and the European Respiratory Society (ERS), endorsed by the International Society of Heart and Lung Transplantation (ISHLT).: the Task Force for the Diagnosis and Treatment of Pulmonary Hypertension of the 
European Society of Cardiology (ESC) and the European Respiratory Society (ERS), endorsed by the International Society of Heart and Lung Transplantation (ISHLT). Eur Heart J 2009; 30: 2493-2537.

6 Galiè N, Hoeper MM, Humbert M, et al. Guidelines for the diagnosis and treatment of pulmonary hypertension. Eur Respir J 2009; 34: 1219-1263.

7 Berger RM, Beghetti M, Humpl T, et al. Clinical features of paediatric pulmonary hypertension: a registry study. Lancet 2012; 379: 537-546.

8 van Loon RL, Roofthooft MT, van Osch-Gevers M, et al. Clinical characterization of pediatric pulmonary hypertension: complex presentation and diagnosis. J Pediatr 2009; 155: 176-182 el.

9 Barst R, Berman Rosenzweig E. Pediatric pulmonary hypertension: screening, diagnosis and assessment. In: M B, ed. Pediatric Pulmonary Hypertension. Munich, Elsevier, 2011; pp. 113-129.

10 Tissot C, Ivy DD, Beghetti M. Medical therapy for pediatric pulmonary arterial hypertension. J Pediatr 2010; 157 : 528-532.

11 Mullen MP. Diagnostic strategies for acute presentation of pulmonary hypertension in children: particular focus on use of echocardiography, cardiac catheterization, magnetic resonance imaging, chest computed tomography, and lung biopsy. Pediatr Crit Care Med 2010; 11: Suppl. 2, S23-S26.

12 Rosenzweig EB, Feinstein JA, Humpl T, et al. Pulmonary arterial hypertension in children: diagnostic work-up and challenges. Prog Pediatr Cardiol 2009; 27: 4-11.

13 Simonneau G, Galiè N, Rubin LJ, et al. Clinical classification of pulmonary hypertension. J Am Coll Cardiol 2004; 43: Suppl. 12, 5S-12S.

14 Jegier W, Sekelj P, Auld PA, et al. The relation between cardiac output and body size. Br Heart J 1963; 25: 425-430.

15 Madani MM, Wittine LM, Auger WR, et al. Chronic thromboembolic pulmonary hypertension in pediatric patients. J Thorac Cardiovasc Surg 2011; 141: 624-630.

16 Jaklitsch MT, Linden BC, Braunlin EA, et al. Open-lung biopsy guides therapy in children. Ann Thorac Surg 2001; 71: 1779-1785.

17 McGoon MD, Miller DP. REVEAL: a contemporary US pulmonary arterial hypertension registry. Eur Respir Rev 2012; 21: 8-18.

18 Peacock AJ, Murphy NF, McMurray JJ, et al. An epidemiological study of pulmonary arterial hypertension. Eur Respir J 2007; 30: 104-109.

19 Stricker H, Domenighetti G, Popov W, et al. Severe pulmonary hypertension: data from the Swiss Registry. Swiss Med Wkly 2001; 131: 346-350.

20 McGoon MD, Krichman A, Farber HW, et al. Design of the REVEAL registry for US patients with pulmonary arterial hypertension. Mayo Clin Proc 2008; 83: 923-931.

21 Badesch DB, Raskob GE, Elliott CG, et al. Pulmonary arterial hypertension: baseline characteristics from the REVEAL Registry. Chest 2010; 137: 376-387.

22 Humbert M, Sitbon O, Chaouat A, et al. Pulmonary arterial hypertension: in France: results from a national registry. Am J Respir Crit Care Med 2006; 173: 1023-1030.

23 Jing ZC, Xu XQ, Han ZY, et al. Registry and survival study in chinese patients with idiopathic and familial pulmonary arterial hypertension. Chest 2007; 132: 373-379.

24 Thenappan T, Shah SJ, Rich S, et al. USA-based registry for pulmonary arterial hypertension: 1982-2006. Eur Respir J 2007; 30: 1103-1110.

25 Taylor CJ, Derrick G, McEwan A, et al. Risk of cardiac catheterization under anaesthesia in children with pulmonary hypertension. Br J Anaesth 2007; 98: 657-661.

26 Carmosino MJ, Friesen RH, Doran A, et al. Perioperative complications in children with pulmonary hypertension undergoing noncardiac surgery or cardiac catheterization. Anesth Analg 2007; 104: 521-527.

27 Hill KD, Lim DS, Everett AD, et al. Assessment of pulmonary hypertension in the pediatric catheterization laboratory: current insights from the Magic registry. Catheter Cardiovasc Interv 2010; 76: 865-873.

28 Hoeper MM, Lee SH, Voswinckel R, et al. Complications of right heart catheterization procedures in patients with pulmonary hypertension in experienced centers. J Am Coll Cardiol 2006; 48: 2546-2552.

29 Feltes TF, Bacha E, Beekman RH 3rd, et al. Indications for cardiac catheterization and intervention in pediatric cardiac disease: a scientific statement from the American Heart Association. Circulation 2011; 123: 2607-2652.

30 Lammers AE, Diller GP, Odendaal D, et al. Comparison of 6-min walk test distance and cardiopulmonary exercise test performance in children with pulmonary hypertension. Arch Dis Child 2011; 96: 141-147.

31 Barst RJ, Ivy DD, Gaitan G, et al. A randomized, double-blind, placebo-controlled, dose-ranging study of oral sildenafil citrate in treatment-naive children with pulmonary arterial hypertension. Circulation 2012; 125: 324-334.

32 Yetman AT, Taylor AL, Doran A, et al. Utility of cardiopulmonary stress testing in assessing disease severity in children with pulmonary arterial hypertension. Am J Cardiol 2005; 95: 697-699.

33 Smith G, Reyes JT, Russell JL, et al. Safety of maximal cardiopulmonary exercise testing in pediatric patients with pulmonary hypertension. Chest 2009; 135: 1209-1214. 